\title{
Simulex; Developing New Computer Modelling Techniques for Evaluation
}

\author{
PETER A. THOMPSON and ERIC W. MARCHANT \\ Unit of Fire Safety Engineering \\ University of Edinburgh \\ Kings Buildings, Mayfield Road, Edinburgh EH9 3JL
}

\section{ABSTRACT}

Many computer models for evacuation have been developed over the past fifteen years, primarily in the form of network-node analyses. These models, by their nature, are severely restricted and cannot accommodate geometrical complexity or compound irregularities in crowd transit. If modern buildings are to be analysed accurately in terms of their evacuation characteristics, then more sophisticated simulation techniques must be adopted. This papers details the development of a computer program that is based on new methods for modelling the motion of individual people in a building. The program makes use of the power of modern P.C.s and is based upon complex spatial analysis and computer-generated route-finding techniques. Particular emphasis is placed upon the incorporation of real-life data into the basic structure of algorithms, and how the performance of the program relates to the design codes[1] and Technical Standards [2].

\section{KEYWORDS:}

Movement, simulation, speeds, parameters, spatial analysis, contours, overtaking, behaviour, validation.

\section{INTRODUCTION}

The development of SIMULEX began by identifying the shortfalls of some of the computer programs developed over the past fifteen years. Network-node models such as EVACNET + [3] made use of simple flow parameters of crowd motion. The user defined a number of rectangular spatial blocks and connecting arcs that modelled rooms and 
pathways in a building. Crowds were treated as homogenous masses, in terms of speed or flow rate, and various assumptions were made about their movement. Behavioural analyses, such as EXITT [4] adopted a different approach by assigning individual speeds, and concentrating much more on the psychological aspects of escape, by using rules formulated by the programmer. These early behavioural models made similar assumptions about the building space, by dividing the building into discrete spatial blocks. The networking of large rectangular spatial blocks may be satisfactory for buildings with simple floor plan layouts, but modern shopping malls, office blocks and stadia are becoming ever more complex and their spatial configurations cannot be considered as individual areas connected by pathways in a network of rooms. The network-node approach requires a high degree of user-input and cannot accommodate obstructions or highly irregular shapes within the building plan.

The most advanced form of the network models in the U.S. is EXIT 89 [5]. This was the first program to achieve the processing of large numbers of individual people and incorporates some modelling of the effect of smoke spread upon the occupants. Within each rectangular space all occupants are assigned the same walking speed, unless impeded by smoke. This model has been incorporated into the HAZARD1 package [6], in an attempt to combine the simulation of fire growth, smoke spread, fire detection, and evacuation. Some degree of correlation with a real-life evacuation of a multi-storey building has been achieved. The program is still under development.

Two programs that have recently been developed in the U.K. use the network-node analysis technique, but on a much finer scale. EGRESS [7] applies certain rules and variables to each person. The building space is represented by a hexagonal grid system. Each hexagonal node represents the volume space of one person, and may be 'occupied' or 'empty'. Each person steps from one position to the next depending on certain rules, and speed is affected by the density of the surrounding crowd. The system is being developed for multi-storey buildings and it is intended that validation against real-life experiments will be carried out as part of the development process. EXODUS [8] is primarily intended for use on mass transport vehicles and the environment space is represented by a network of nodes, connected by arcs that define the available path of movement from one position to the next. Each node represents a small spatial area, and is regarded as either occupied or empty. Many character traits are described, and input from a fire / smoke spread program can be received and used to affect the behaviour of the occupants. An early validation exercise was executed by comparing EXODUS output to some aircraft evacuation tests and the experimental trends were simulated. Some of the simulated evacuation times were similar to the real-life tests, but not consistently so. This was possibly due to the lack of specific data for the traits of individuals in each evacuation.

The VEGAS system [9] has arisen from the recent developments in virtual reality techniques. The system illustrates fire growth, smoke spread, and occupant behaviour using real-time animation. The psychological aspects modelled are; group behaviour, alarm awareness and the effects of smoke. Each person chooses a direction by assessing userspecified target points along alternative escape routes. Escape only starts after the necessary threat triggers, such as alarm and smoke have been perceived. Unlike other models, this system does not use the commonly referenced speed / density curves, but instead looks at proximity logic, the mutual obstruction of escaping people and forces acting on their bodies at crowded doorways. One of the primary advantages of this system 
is its ability to communicate the building environment to the user, due to the clarity of the 3-dimensional graphics.

All of these models require a high degree of user-input. Throughout the development of SIMULEX the authors have attempted to minimise user-inputs while increasing the complexity and number of the computer algorithms in the program. EGRESS [7] and EXODUS [8] both use algorithms that set up a value of potential distance to exit at each node, but the directions of movement depend upon the number of arcs connecting one cell to another. This is however, a significant improvement upon the earlier network models, due to the resolution of the grids involved, and creates more spatial accuracy with less numerical user-inputs.

\section{PRIMARY OBJECTIVES OF SIMULEX}

A number of definite objectives were formulated at the outset of this project. They dictated the basic structure of the model and determined the underlying approach to solving problems that arose. The program sets out to:

(i) Define the building space with only the building plan as a user-input.

(ii) Input the building plan using graphical techniques, and a 'mouse' in order to reduce the time taken to define the building space. $\mathrm{CAD}$ package outputs should eventually complement this process.

(iii) Automatically define both the building space and escape routes for any building geometry, with a high degree of accuracy. Flexibility should also be incorporated in order that the user can change the nature of the escape routes, if desired.

(iv) Define individuals by creating a stack of person characteristics and co-ordinates using inputs based on population density figures from the Technical Standards [2] or research data. There will be a random distribution of occupant characteristics such as age and walking speed.

(v) Incorporate flexibility into the definition of escape routes, so that overtaking and similar behaviour consisting of localised route deviation can occur.

(vi) Incorporate speed reduction effects due to the proximity of other individuals; overtaking and 'jostling' algorithms, and floating point arithmetic for the assessment of angles of orientation and position.

(vi) Produce a plan view of the evacuation process, displaying a clock and a graphical representation of each person's movement with the passage of time. 


\section{TifE SUITE OF PROGRAMS IN THIS PROJECT}

The following programs were written to contribute to this project;

(i) DRAWPLAN - allows the user to define the building geometry.

(ii) GRIDFORM - handles output from DRAWPLAN and segments the building space into a fine mesh of spatial squares. Once this is done, the routines create a map of boundary exits and distance contours throughout the building space.

(iii) ONEMAN - uses data from DRAWPLAN and GRIDFORM, and uses this data to animate one person's movement from the most remote point in the building to the nearest building exit.

(iv) SIMULEX - uses data from DRAWPLAN, GRIDFORM, and user-specified inputs describing population characteristics. A simulation of the building evacuation is then animated and analysed.

\section{DRAWING THE BUILDING PLAN WITH 'DRAWPLAN'}

The graphics program DRAWPLAN allows the user to draw in the building plan using the 'mouse' and a few key controls. Speed of input is obtained by creating walls and obstructions by defining rectangular 'building blocks', of any orientation and width. Only two 'clicks' of the mouse are required to draw a solid wall, so that the plan may be input as quickly and easily as possible. It is envisaged that the final version of this program will handle CADD outputs as an option.

\section{DEFINING SPACE AND POTENTIAL EXIT ROUTES WITH 'GRIDFORM'}

This program processes the data from DRAWPLAN and automatically forms a high definition integer distance grid where all walls or solid objects are assigned the maximum value, and exit values are set to zero. Within this grid the program sets up a fine mesh of data points whose assigned values are equal to their geometrical distance to the nearest exit. The plan area that each data point represents is equal to 0.0625 square metres.

The optimum route to exit from any point can be evaluated by assessing the pattern of surrounding values. The easiest way of visualising the route-finding system is to represent the 'spatial mesh' values in bands, as contours of distance from the nearest exit. The shortest route to exit at any point is obtained by heading at right angles through the contours. The distance contour map shown in Figure 1 is set up so that the optimum direction to the nearest exit is accurate to within 2 degrees, while computer processing time remains relatively short. The system is fairly flexible. Before the mesh is formed, the values at exits can be altered (instead of automatically set to zero) and this affects the whole pattern of the final 'distance map'. If an exit value is increased before the 'distance map' is formed, a bias is created, making exits more or less desirable. Research has proved that occupants often prefer to evacuate through more familiar escape routes, but comprehensive data is not yet available. 


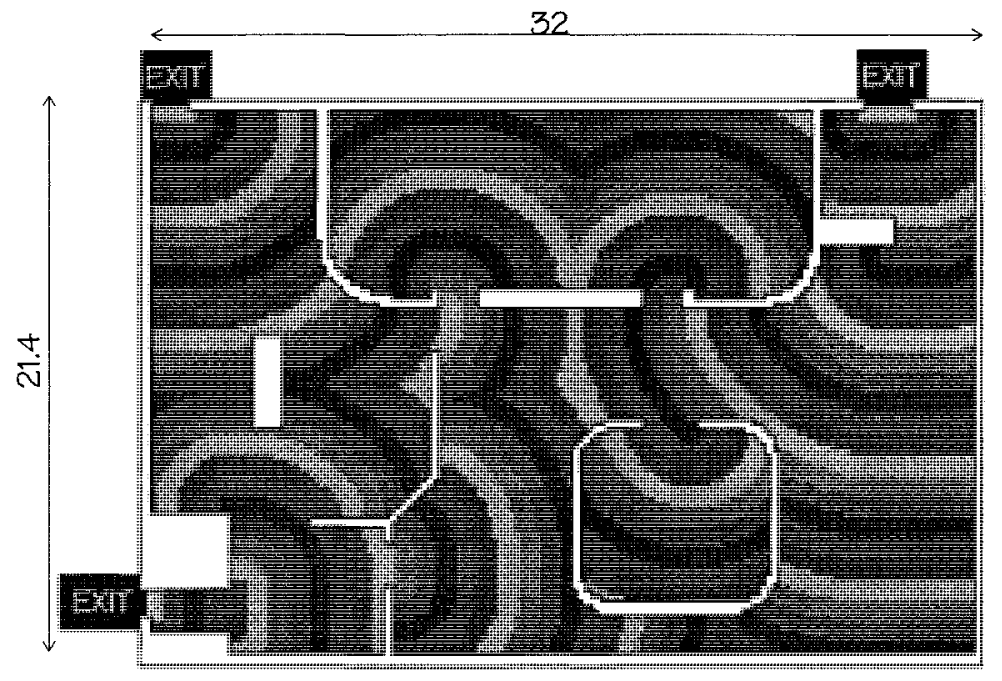

Dims. $(\mathrm{m})$. Walls / obstructions in white with black outline. 1 Contour Width $=0.8 \mathrm{~m}$

Figure 1. Distance Contour Map for a sample office space.

\section{ASSESSING TRAVEL DISTANCE WITH 'ONEMAN'}

This program uses the building plans and contour maps already formed. The point that is most remote from any exit is automatically identified by the computer. The route taken by a single person from this point to the nearest exit is then animated, with the time and distance travelled displayed as motion progresses. The final output displays the total route, the time taken and the distance travelled. The distance travelled is equal to the 'travel distance' as defined by the design codes [1]. The angle of travel is accurate to two degrees. Accuracy can be increased, if desired, by increasing the area of the scanning arrays used to set up the distance contour map.

\section{MODELLING EVACUATION WITH 'SIMULEX'}

The main program, SIMULEX, uses the building plan data compiled by 'DRAWPLAN' and 'GRIDFORM'. On execution, the program requests the name that has been assigned to the data files for one building, and then loads them. The building plan is displayed with a dialogue box at the bottom of the screen. The user is asked firstly if 'movement traces' are desired, and secondly about details of the population densities. If 'movement traces' are selected then individuals leave coloured trails behind them as they move, creating a graphical display of all of the escape routes taken. If 'movement traces' are not selected, then individual movement is animated, and the lines of individual escape routes are not displayed. The population density is input by specifying one density over the 
whole building, or different densities for different areas. The program then begins initialising the simulation.

\subsection{POPULATION CHARACTERISTICS}

At present, SIMULEX assumes that the building population consists of people with ages randomly distributed between 12 and 55, with male and female genders. Thus, the unimpeded walking speeds of individuals vary from 0.8 to $1.7 \mathrm{~m} / \mathrm{s}$, and are assigned automatically when the stack of population characteristics is formed. These figures are based on the data from Ando et al [10], and can be adjusted if different characteristics are desired. The orientation of each individual's body is randomly distributed amongst the population when the 'character stack' is created. The co-ordinate position of each person is defined by creating a regular plan grid of people. The distances between people in this grid are calculated from the densities specified by the user at the outset.

\subsection{RUNNING SIMULEX}

When SIMULEX commences, all the movement algorithms are invoked for each individual, nine times per second. This frequency of assessment was chosen because the fastest person in the system could progress no more than $200 \mathrm{~mm}$ between each movement assessment scan. Individual motion is evaluated by the methods described in Sections 7.2 7.5. The dialogue box displays the time clock, the number of people in the building, and the file name. When the last person leaves the building, the total evacuation time is displayed.

\subsection{ASSESSING ROUTE TO EXIT}

In the first instance, each person uses the contour map to assess the optimum direction to exit (in terms of distance). This is done by looking in the direction that is perpendicular to the distance contours at that position. If the speed algorithm decides that motion is potentially impeded in this direction, then the overtaking and jostling algorithms are invoked.

\subsection{FLUCTUATIONS IN INDIVIDUAL WALKING SPEEDS}

The essence of simple crowd flow is the relationship between the proximity of individuals and their walking speed. As the distance between people in a crowd is reduced, the crowd density increases and the overall speed of forward motion in the group decreases. When the proximity increases to such a degree that bodily contact is incurred, then 'shuffling' and further irregular speed reduction occurs. At very high crowd concentrations 'body arching' or jamming can cause major speed reductions, and halt the forward motion of the crowd. This pattern of beha viour has been observed and collated by many researchers, some of whose results are summarised in Figure 2. Note that the units are those used by Ando et al [10], and the other data values from Fruin [11], Hankin \& Wright [12] and Predtechenskii \& Milinskii [13], have been converted from different units. 


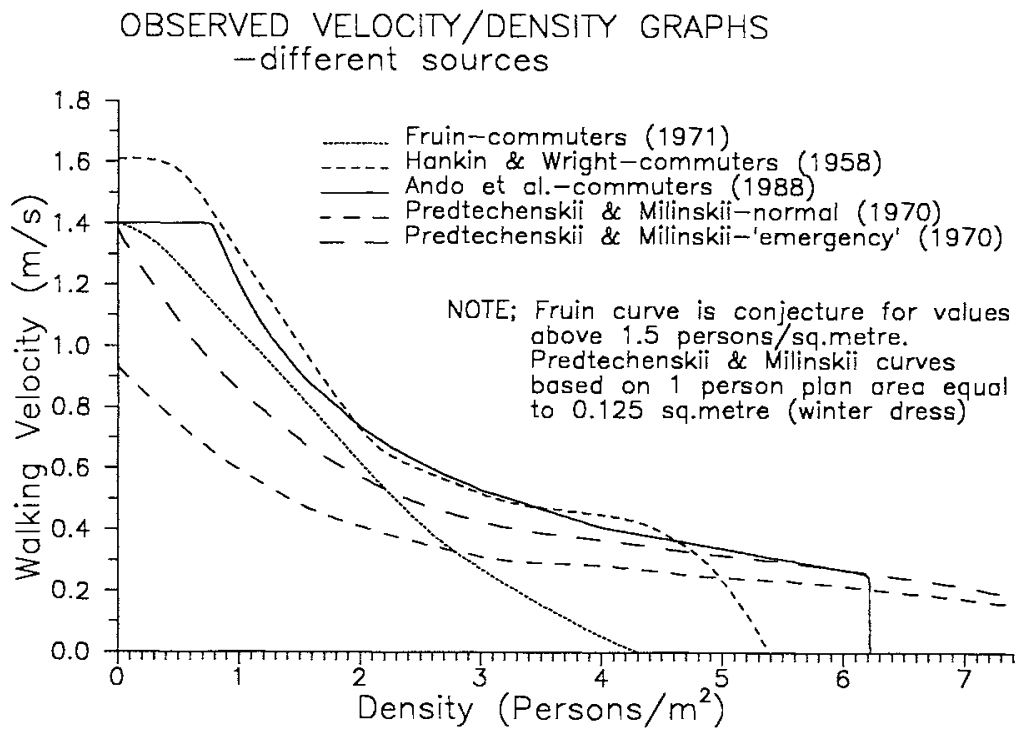

Figure 2. A comparison of available data on the crowd velocity / density relationship.

Speed reductions due to the proximity of others are calculated using an approximation of the speed / density curve presented by Ando et al [10]. Unfortunately, the curve assesses speed in relation to crowd density over a given area, so it is necessary to convert the data to a form that relates speed to distances between individuals. SIMULEX requires data in this form in order that individual movement can be evaluated.

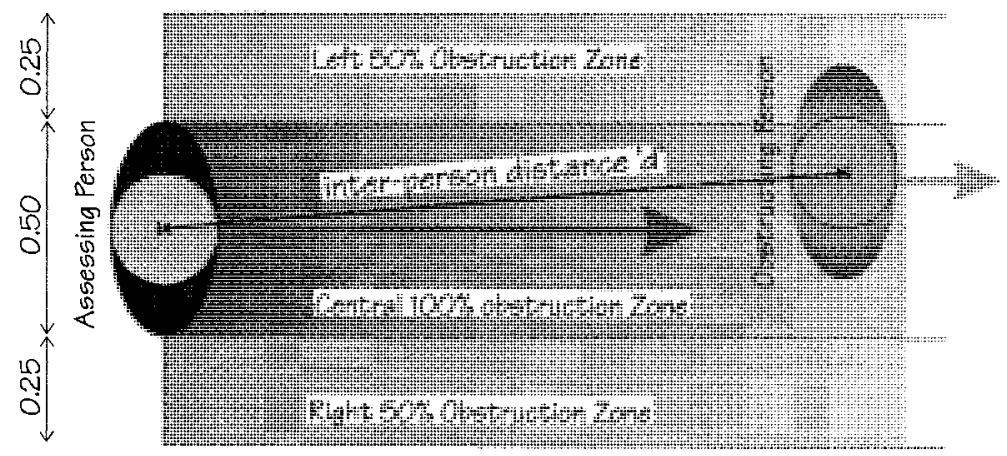

Forward Path

Figure 3. Obstruction Zones For Speed Assessment. 
For the purposes of the program, the speed is related to the inter-person distance ' $d$ ' between centre co-ordinates of the assessing and obstructing persons, as illustrated in Figure 3 .

Fruin [11] and Ando et al [10] observed regular packing characteristics in uniform crowds. Equation (1) is based upon these observations, and assumes that in a crowd of evenly spaced people, the area per person is equal to the square of the average inter-person distance. Using this spatial assumption, the velocity/density relationships described in Figure 1 are approximated in terms of inter-person distance by Equation (2). The graph of the final data, derived from the figures from Ando et $a l[10]$ is shown in Figure 3.

$$
d=\sqrt{\frac{1}{D}}
$$

reduced.speed $=\left(\frac{V(d-0.25)}{0.87}\right)$

Note: $d$ is inter-person distance $(\mathrm{m}), D$ is density (Number of persons $\left./ \mathrm{m}^{2}\right), V$ is unimpeded walking speed $(\mathrm{m} / \mathrm{s})$. NOTE: if $d>1.12$ then speed is assumed to be unimpeded. This 'distance threshold' of $1.12 \mathrm{~m}$ can be adjusted if desired.

The formula is fully applied when the nearest obstructing person is within the $100 \%$ zone (Figure 2). If the nearest obstructing person is within a $50 \%$ zone, the speed reduction is multiplied by 0.5 , and a second 'nearest person' in either of the other two zones will also incur only half of the speed reduction effect.

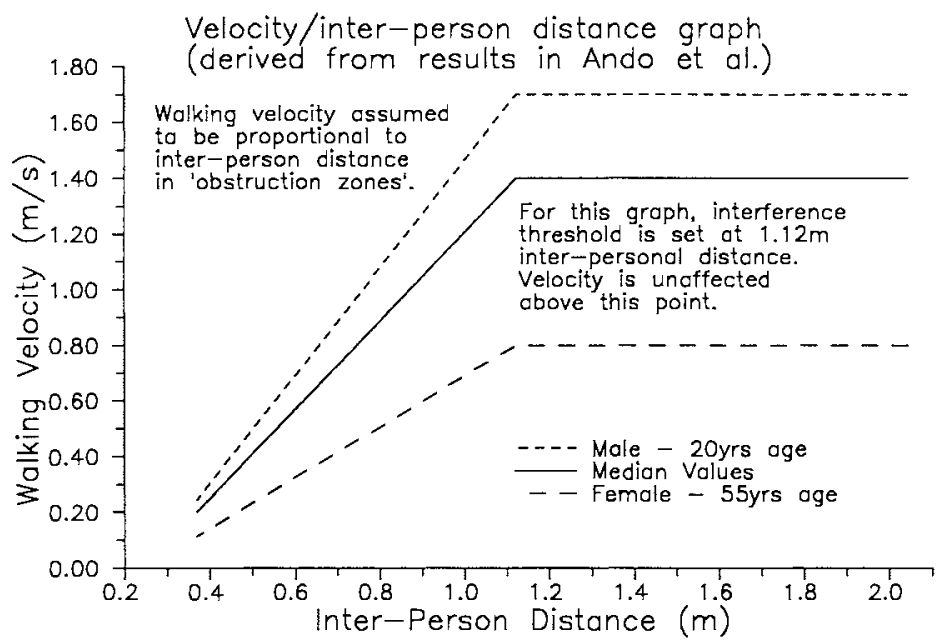

Figure 4. The velocity / distance graph used by SIMULEX 
To check that SIMULEX displays the suitable range of speeds, and associated variation in speed within the population, it is possible to convert the data shown in Figure 4 back into units of density instead of inter-person distance. Thus, the emulated graph of velocity against density was obtained by using Equations (1) and (2) and is illustrated in Figure 5 .

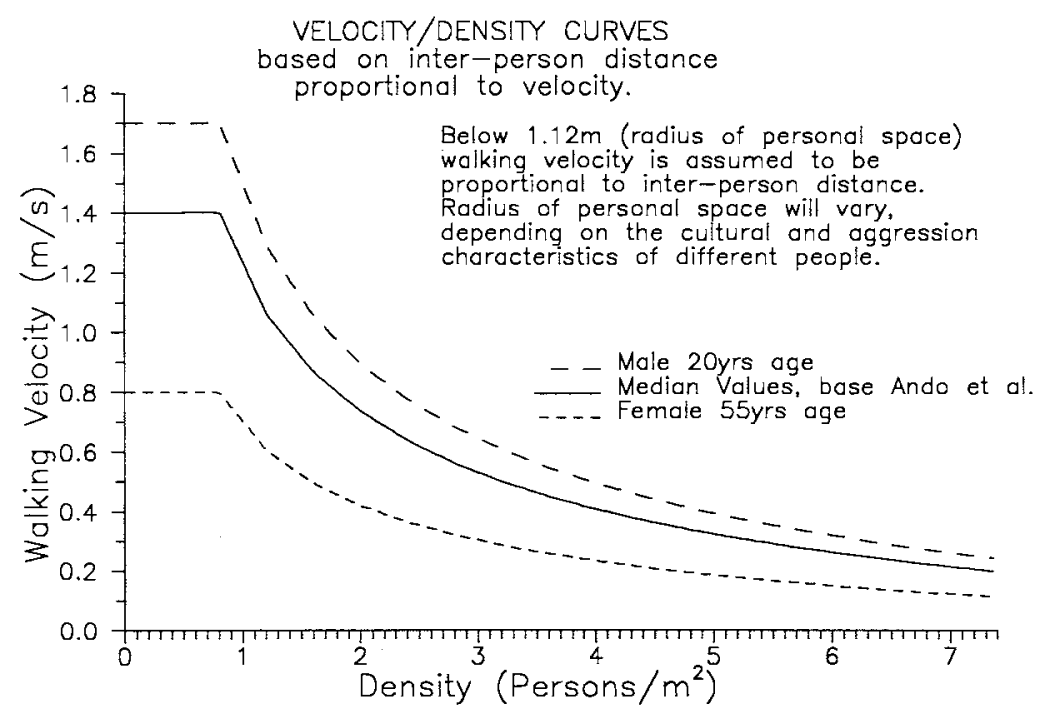

Figure 5 . The velocity / density curves emulated by SIMULEX

\subsection{OVERTAKING AND LOCALISED ROUTE DEVIATION}

If the walking speed of an individual is impaired by the proximity of one or more obstructing persons, then the overtaking routines are invoked. The program scans either side of the direction of travel, and if a significant improvement in speed is obtained, then the direction of travel changes to the new deviated angle. The angle of deviation, or 'overtake angle', increases as an obstructing person becomes closer. Overtaking and localised deviation of route is observed frequently, and the deviation routine produces a degree of jostling in the higher density crowds.

\subsection{MOVEMENT CHARACTERISTICS}

The program, by its nature, models queuing inherently. The speed calculations never yield a zero value, but each person is only allowed to move into unoccupied space and will therefore become stationary if exactly behind another person. Twisting movements of the body are restricted by an 'available twist factor' which is currently set at 0.01 seconds/degree. This twist factor is to be checked and updated, depending on the results of forthcoming observational research. As yet, no pushing or pressure build-ups are modelled. 


\section{EARLY SIMULATION RUNS}

In order to assess the maximum flow capacity of a doorway width, the program was used to model high density, non-panicking crowds at doorways of different widths. With slight changes of the overtaking/jostling algorithm and the order in which speed assessment occurs (scanning from [most remotely positioned person] first, to [person at exit] last, or visa versa) then the flow rate ' $q$ ' can vary from 1.5 to 1.9 persons $/ \mathrm{m} / \mathrm{s}$. Real-life observations of $\mathrm{q}$ include 1.5 ('Fire \& Buildings' [14]) and $1.8 \mathrm{persons} / \mathrm{m} / \mathrm{s}$ (Melinek [15]) This correlation of simulated value to real life observation is encouraging, especially in view of some of the assumptions made in the speed assessment routine.

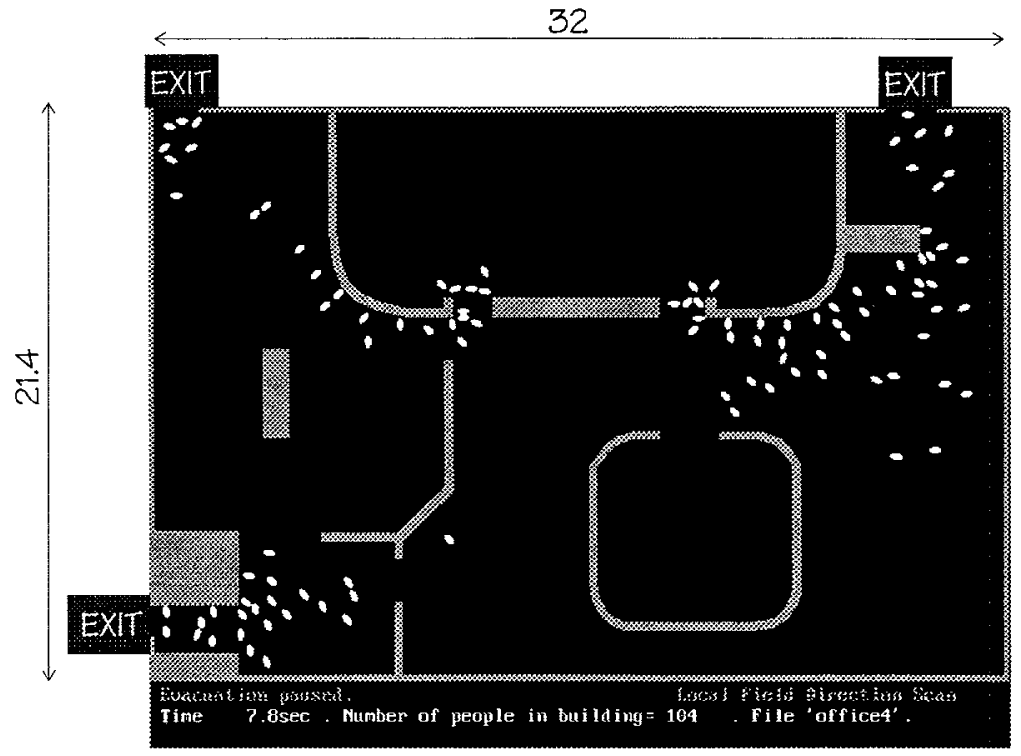

Figure 6. Sample Screen Display for an office space evacuation. Dims.(m).

Figure 6 demonstrates the ability of the program to cope with complex geometries and obstructions of any size. In the example shown, immediate reaction to alarm was assumed. It became clear from watching the progress of various simulations that the presence of a narrow obstruction in the centre of a 1.8 metre wide opening significantly reduced flow capacity, even though the total combined opening width was only reduced by $0.1 \mathrm{~m}$.

\section{CONCLUSIONS AND DEVELOPMENT}

SLMULEX adopts a new approach to modelling the evacuation of buildings. It combines the ease and speed of user-input with geometrical complexity and a population with individual characteristics of movement and speed. The aim is to produce a program that accepts population density values from the design codes [1], or real observational 
measurements, but uses modern research and computing power to evaluate the potential evacuation of a complex building with a high degree of accuracy. At the same, it illustrates to the user any restrictive areas of the building where bottlenecks and 'jams' occur, in order that the design may be adjusted and optimised for the purposes of evacuation.

The route-finding techniques that have been developed are geometrically accurate and 'travel distance' is assessed automatically. A choice of exit can be achieved by overlaying various distance contour maps based on different available final exits, and the effects of smoke spread could be simulated by the progressive addition of weighting factors to the contour maps over increasing areas of the building. At this stage, staircases have not yet been modelled because validation on a single storey, flat plane is required first.

More complex psychological factors affecting the building population are currently under investigation. One important factor is the time taken for individuals to react to an alarm and begin the physical escape process. This 'first stage' of evacuation is affected by an individual's awareness, eagerness to move, and the nature of the alarm. Studies, such as those carried out by Proulx [16] could be used as a basis for numerical values, but the data available at present does little more than suggest that the reaction time to alarms can vary from 10 seconds to 15 minutes. Other complex psychological factors such as the familiarity of routes and aggressiveness will be incorporated in a later version, but additions such as these must require a greater degree of user-input.

The strong correlation between test results and real-life values of crowd flow rate per unit exit width is very important. More large scale validation is required, and observational tests on evacuating people are to be carried out in the spring of 1994 in order that the accuracy of the computer methods can be determined. Results such as overtaking behaviour, twisting ability, and general route deviation will be logged and compared to the performance of the computer algorithms. Any parameters or assumptions found to be inaccurate in any way will be identified and adjusted. The future development of SIMULEX will be based upon combining progressive advancement with the results from real-life observations and tests.

\section{REFERENCES}

1. British Standards Institution (1983), BS 5588: Part 3: 1983, "Fire precautions and the design and construction of buildings: Part 3: Code of Practice for Office Buildings", London, BSI, 1983.

2. The Scottish Office(1990). "Technical Standards for Compliance with the Building Standards (Scotland) Regulations 1990", Edinburgh, HMSO, 1990-1993

3. T. M. Kisko \& R. L. Francis, "EVACNET+: A computer program to determine optimum building evacuation times", Fire Safety Journal, 9, 220, 1985.

4. B. M. Levin, "EXITT - A Simulation Model of Occupant Decisions and Actions in Residential Fires", in Fire Safety Science - Proceedings of the Second International Symposium, International Association of Fire Safety Science, Tokyo, June 13-17, 1988, Hemisphere Publishing Company, New York. 
5. R.F. Fahy, "EXIT 89: An Evacuation Model for High-Rise Buildings", in Fire Safety Science - Proc. of the 3rd Int. Symp. of Fire Safety Science, University of Edinburgh, July 8-12, 1991, Elsevier Science Publishers, London 1991, pp 815823.

6. R.W. Bukowski, W.W. Jones, B.M. Levin, C.L. Forney, S.W. Steifel, V. Babrauskas, E. Braun, and A.J. Fowell, "HAZARD I - Volume 1: Fire Hazard Assessment Method ", National Institute of Standards and Technology Center for Fire Research, NBSIR 87-3602, July 1987.

7. N.Ketchell, D.M. Webber, C.A. Marriot, P.J. Stephens, I.R. Brearley, J.G Fraser, J. Doheny, J. Smart, "The EGRESS code for human movement and behaviour in emergency evacuations, in R.A. Smith and J.F.Dickie (eds) "Engineering for Crowd Safety", Elsevier Applied Science, Amsterdam, 1993.

8. E.R. Galea, J.M. Perez Galparsoro and J.Pearce, "A Brief Description of The EXODUS Evacuation Model", 18th Internat. Conf. Fire Safety, San Francisco, 1993

9. K. Still, "New computer system can predict human behaviour response to building fires.", 'Fire', (84) pp 40-41, Jan. 1993.

10. K. Ando, H. Ota \& T. Oki, "Forecasting the Flow of People", (in Japanese), R.R.R. Railway Research Review, (45). 8, 1988, pp 8-14.

11. J. Fruin, "Pedestrian Planning and Design", Metropolitan Association of Urban Designers and Environmental Planners, New York, 1971 (out of print).

12. B.D. Hankin \& R.A. Wright, "Passenger Flows in Subways", Operational Research Quarterly, Vol. 9, pp 81-88.

13. V.M. Predtechenskii \& A.I. Milinskii, "Planning for Foot Traffic Flow in Buildings", Amerind Publishing Co.Pvt.Ltd., New Delhi, 1978 (translated from Russian) originally published in 1970.

14. The Aqua Group, "Fire and Buildings", Granada Publishing Ltd., London 1984.

15. S.J. Melinek. \& S. Booth, "An analysis of evacuation times and the movement of crowds in buildings", Building Research Establishment Current Paper CP96/75 (October 1975), Building Research Establishment, Fire research Station, Borehamwood, Herts.

16. G. Proulx \& J.D. Sime. "To Prevent Panic in an Underground Emergency: Why not tell people the truth ?", Proc. of the 3rd Int. Symp. of Fire Safety Science, University of Edinburgh, July 8-12 ,1991, Elsevier Science Publishers, London 1991. 